Relations industrielles

Industrial Relations

\title{
L'industrie de la chaussure
}

\section{Gérard Tremblay}

Volume 5, numéro 5, février 1950

URI : https://id.erudit.org/iderudit/1023322ar

DOI : https://doi.org/10.7202/1023322ar

Aller au sommaire du numéro

Éditeur(s)

Département des relations industrielles de l’Université Laval

ISSN

0034-379X (imprimé)

1703-8138 (numérique)

Découvrir la revue

Citer cet article

Tremblay, G. (1950). L'industrie de la chaussure. Relations industrielles /

Industrial Relations, 5(5), 43-46. https://doi.org/10.7202/1023322ar

Tous droits réservés (C Département des relations industrielles de l’Université Laval, 1950
Ce document est protégé par la loi sur le droit d'auteur. L'utilisation des services d'Érudit (y compris la reproduction) est assujettie à sa politique d'utilisation que vous pouvez consulter en ligne.

https://apropos.erudit.org/fr/usagers/politique-dutilisation/ 


\section{L'INDUSTRIE DE LA CHAUSSURE}

\section{Gérard Tremblay}

Le dernier rapport annuel du Comité paritaire de l'industrie de la chaussure, couvrant la période du ler septembre 1948 au 31 août 1949, nous fournit une multitude d'informations précieuses inédites tant au point de vue de la production que des relations patronales-ouvrières.

Pour notre province du moins où le Comité a juridiction, il établit de façon précise le nombre d'unités productrices dans chaque zone territoriale déterminée par le décret relatif à cette industrie, le nombre d'ouvriers qui y sont employés et les salaires payés.

\section{La production}

Les statistiques du Département du commerce d'Ottawa établissent qu'en 1948 la production totale des paires de chaussure atteint pour le $\mathrm{Ca}$ nada 32,326,766; pour sa part, Québec s'en est assuré $19,580,637$, soit $60.5 \%$, et l'Ontario, 12,048 ,679 , soit $37.2 \%$.

En 1937, soit deux ans avant la deuxième guerre, la production canadienne atteignait 24,325,355 paires, celle de Québec, 15,462,603, soit $63.56 \%$, et celle de l'Ontario, $8,357,412$, soit $34.35 \%$.

La valeur de cette production, pour tout le Canada, était, en 1948 , de $\$ 104,665,327.00$, la part du Québec étant de $\$ 63,533,871.00$, soit $60.7 \%$, et celle de l'Ontario, $\$ 36,973,103.00$, soit $35.3 \%$.

En 1937, la valeur de la production canadienne n'était que de $\$ 40,905,216.00$, celle du Québec atteignant $\$ 24,772,574.00$, soit $60.5 \%$, et celle de l'Ontario, $\$ 14,597,082.00$, soit $35.5 \%$.

On conclut facilement de là que Québec et l'Ontario contrôlent au delà de $95 \%$ de la production, soit en nombre de paires, soit en valeur, dans l'industrie canadienne de la chaussure, Québec comptant pour près des deux tiers et l'Ontario pour un tiers.

Si l'on étudie les statistiques pour la décade s'étendant de 1938 à 1948, on constatera que cette proportion dans les chiffres respectifs de la production des deux provinces industrielles se maintient sans écart appréciable.

\section{Le décret}

Les relations patronales-ouvrières dans l'industrie de la chaussure sont stabilisées depuis une assez longue période, soit une quinzaine d'années, alors que des conventions collectives rendues obligatoires par décrets sous la Loi du même nom ont été négociées entre organisations patronales et ouvrières. Les premières conventions avaient comme signataires, du côté patronal, des manufacturiers individuels et l'Association des manufacturiers de chaussures de Québec; du côté ouvrier, quelques syndicats de l'industrie de la chaussure. Plus tard on vit apparaître l'Association rurale des manufacturiers de chaussures comme co-signataire.

Les employeurs, sous la pression de la nécessité des négociations collectives, ont formé depuis quelques années l'Association patronale des manufacturiers de chaussures du Québec, laquelle englobe pratiquement tous les manufacturiers de la province. Du côté ouvrier, le pluralisme syndical s'est maintenu et on note comme signataires la Fédération nationale du cuir et de la chaussure du Canada inc., «The Boot and Shoe Workers' Union 》 et l'Association des travailleurs en chaussures inc.

La juridiction industrielle du décret est déterminée par l'article I de l'arrêté-en-Conseil \#3003, du 31 juillet, 1946, qui se lit comme suit:

«L'industrie de la chaussure comprend la fabrication, pour le tout ou partie, de chaussures de tous genres, de toutes descriptions, de tout matériel, que cette fabrication constitue ou non le commerce principal de l'entreprise ou un commerce secondaire; elle comprend aussi la fabrication ou la confection du matériel utilisé dans la fabrication de la chaussure dans tout établissement industriel lui faisant subir une opération quelconque ».

Le décret couvre toute la province; toutefois, il a fallu tenir compte des conditions économiques des diverses régions pour établir les taux de salaires comme l'implique du reste l'article 6 de la Loi de la convention collective. La province est donc divisée en trois zones:

Zone I: L'Ile de Montréal et le territoire compris dans un rayon de cinq (5) milles autour de ses limites;

Zone II: La Cité de Québec et le territoire compris dans un rayon de cinq (5) milles autour de ses limites; 
Zone III: Toute la province de Québec, à l'exception des Zones I et II.

Les taux de salaires comportent entre les Zones I et II un décalage de $5 \%$, entre les Zones I et III, de $15 \%$.

Le décret comporte six (6) classes d'ouvriers. Les taux honoraires minima pour les salariés travaillant à la pièce sont les suivants:

$\begin{array}{cccc}\text { Classe } 1 & \begin{array}{c}\text { (Zone I) } \\ \$ 1.045\end{array} & \begin{array}{c}\text { (Zone II) } \\ \$ 0.99\end{array} & \begin{array}{c}\text { (Zone III) } \\ \$ 0.915\end{array} \\ \text { Classe } 2 & .88 & .835 & .77 \\ \text { Classe } 3 & .77 & .73 & .67 \\ \text { Classe } 4 & .605 & .57 & .53 \\ \text { Classe 5 } & .44 & .42 & .385 \\ \text { Classe 6 } & .385 & .365 & .335\end{array}$

Ces deux dernières classes comprennent évidemment des opérations ne requérant qu'un minimum de compétence.

\section{Distribution des ateliers}

Au ler septembre, 1949, la province comptait 207 fabriques de chaussures: Zone I, 88; Zone JI, 34; Zone III, 85.

Il est intéressant de noter la variation du nombre d'ateliers, par zone, depuis 1936. Cette variation peut nous révéler si le décret a été un facteur de stabilisation dans l'industrie ou si, au contraire, il a favorisé davantage la région de Montréal, celle de Québec ou les zones rurales proprement dites. Voici le mouvement dans la distribution géographique des ateliers pour la période de 1936-37 à 1948-49:

$\begin{array}{lcccc} & \text { Zone I } & \text { Zone II } & \text { Zone III } & \text { Total } \\ 1936-37 & 64 & 33 & 52 & 149 \\ 1937-38 & 71 & 41 & 55 & 167 \\ 1938-39 & 69 & 33 & 56 & 158 \\ 1939-40 & 66 & 29 & 54 & 149 \\ 1940-41 & 71 & 26 & 52 & 149 \\ 1941-42 & 74 & 27 & 46 & 147 \\ 1942-43 & 69 & 24 & 47 & 140 \\ 1943-44 & 68 & 24 & 48 & 140 \\ 1944-45 & 70 & 27 & 62 & 159 \\ 1945-46 & 87 & 31 & 79 & 197 \\ 1946-47 & 93 & 34 & 97 & 224 \\ 1947-48 & 93 & 37 & 86 & 216 \\ 1948-49 & 88 & 34 & 85 & 207\end{array}$

Nous l'avons déjà indiqué, la Zone I comprend l'Ile de Montréal et un rayon de cinq (5) milles; la Zone II, Québec et un rayon de cinq (5) milles; la Zone III, le reste de la province.

Il est intéressant de noter qu'au point de vue territorial la Zone III couvre trente-neuf (39) mu- nicipalités qui sont situées dans les Cantons de l'Est, dans la Vallee de la Chaudière, dans la Vallée du St-Maurice et même au Lac St-Jean. ${ }^{1}$

\section{Marché du travail}

Le nombre et la situation des ateliers n'indiquent pas l'importance de leur production; pour l'apprécier il faut recourir plutôt à la distribution numérique de la main-d'oeuvre. En 1949 l'industrie comptait 16,051 employés dont 13,285 possédant un emploi régulier: la Zone I, 8,694 employés dont 4,270 hommes et 3,974 femmes; la Zone II, 3,367 dont 1,808 hommes et 1,559 femmes; la Zone III, 3,990 dont 2,151 hommes et 1,839 femmes. Ces statistiques établissent que $45 \%$ des employés sont du sexe féminin. Elles révèlent de plus que la plus grosse partie de la production, soit un peu plus de $50 \%$, s'exécute à Montréal, la région de Québec et les zones rurales se partageant l'autre moitié.

Le rapport du Comité fait un regroupement des ateliers d'après le nombre d'employés pour chaque zone et en subdivisant ces ateliers en petits établissements employant de 1 à 10 ouvriers, en établissements moyens employant de 10 à 25 ouvriers et en établissements employant $25 \mathrm{ou}$ vriers et plus. Voici le tableau de cette répartition que nous commentons dans nos conclusions:

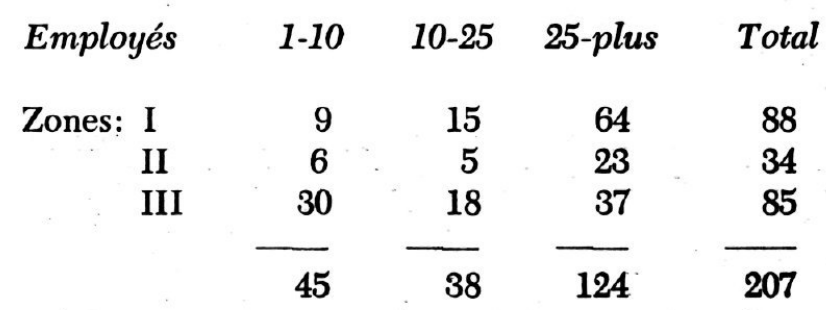

\section{Conditions de travail}

Nous limitons nos observations à la durée du travail et aux salaires. La durée moyenne hebdomadaire du travail pour l'année 1948-49 a été de 32.5 heures. Quant au décret, il établit à l'article 21 une semaine de travail maximum de quarante-huit (48) heures avec paiement de salaire et demi pour tout travail supplémentaire. Cependant, comme l'immense majorité des ouvriers travaillent sur une base de salaire à la pièce les res-

(1) Luceville, Trois-Pistoles, Roberval, Chicoutimi, Bagotville, Lac St-Charles, Notre-Dame des Laurentides, Giffard, Loretteville, St-Emile, Village Huron, Sillery, Qué., Ste-Marie, Valley Jct., Beauceville, St-Georges, St-Samuel, Lac Mégantic, Sherbrooke, Richmond, Victoriaville, Plessisville, St-Tite, Grand'Mère, SteGeneviève, Trois-Rivières, Lavaltrie, Contrecoeur. Upton, Acton Vale, St-Joseph de Yamaska, St-Hyacinthe, St-Pie. Marieville, St-Césaire, St-Rémi, Terrebonne, St-Jérôme, L'Assomption. 
trictions à la durée du travail n'opèrent que dans certains cas particuliers. Au surplus, comme nous le disons plus haut, la durée effective et moyenne de la semaine de travail n'a été pour l'année écoulée que de 32.5 heures. De 1937-38 à 1948-49 cette durée hebdomadaire du travail s'établit comme suit:

\begin{tabular}{|c|c|c|}
\hline $1937-38$ & .. $\ldots . . .29 .75$ & 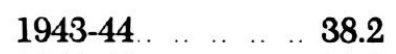 \\
\hline 1938-39. & $\begin{array}{llll}\text {.. } & . . & & 31.5\end{array}$ & $1944-45$. \\
\hline $1939-40$ &.. .34 & $1945-46$ \\
\hline $1940-41$. & .. 38 & $1946-47$. \\
\hline 1941-42 & 38.9 & $1947-48$ \\
\hline $1942-43$ & 41.8 & $1948-49$ \\
\hline
\end{tabular}

On se rend compte que l'industrie a connu son maximum de rendement en l'an 1942-43 avec une semaine moyenne de 41.8 heures. Le travail a été presque également distribué dans les trois zones pour l'année $1948-49$, la moyenne par semaine ayant été de 32.8 heures dans la Zone I, de 31.3 heures dans la Zone II et de 33 heures dans la Zone III.

Quant aux salaires, le total pour la province a été de $\$ 15,973,461.09$ - Les salaires payés dans la Zone I ont été de $\$ 9,673,842.24$; dans la Zone II, $\$ 2,726,938.11$; dans la Zone III, $\$ 3,572,680.74$.

Inutile de dire que depuis 1937-38 le progrès du point de vue salaire global a été constant tant en raison de l'augmentation du nombre des ouvriers et de la production que de l'accroissement du taux de la rémunération proprement dite. Le rapport du Comité a fixé pour 1937-38 un nombre indice de 100; pour les années subséquentes, voyons quel a été le mouvement de l'indice du salaire global:

\begin{tabular}{|c|c|}
\hline $1937-38 \ldots \ldots \ldots 100$ & $1943-44 \ldots \ldots \ldots \ldots .193 .1$ \\
\hline $1938-39 \ldots \ldots \ldots . \quad \ldots 102.7$ & $1944-45$ \\
\hline $1939-40 \ldots \ldots$ & $1945-46$ \\
\hline $1940-41 \ldots$ & $1946-47$. \\
\hline $1941-42$. & $1947-48$ \\
\hline $1942-43 \ldots \ldots . . . \quad .186 .9$ & $1948-49$. \\
\hline
\end{tabular}

Le rapport n'indique pas le salaire moyen des ouvriers et des ouvrières. Ce salaire moyen du reste ne serait pas équitable pour l'industrie étant donné que celle-ci comporte une multitude d'opérations dont les unes requièrent une haute qualification de la main-d'oeuvre alors que d'autres, surtout celles des classes 5 et 6 déterminées par le décret, n'en exigent que très peu.

Le Comité a cru bon cependant d'indiquer le salaire moyen de quelques catégories. Ainsi, pour la Zone I, le salaire des tailleurs de cuir travaillant à la pièce était de $\$ 1.23$ tandis que le taux de ceux travaillant à l'heure était de \$1.049; pour la Zone II, $\$ 1.12$ et .944; pour la Zone III, $\$ 1.048$ et .885 . Le salaire moyen pour toute la province était de $\$ 1.099$.

Le rapport indique les salaires moyens horaires pour les départements de la couture, du cuir à semelles, du montage, fonçage et finition. Pour certaines opérations majeures, le salaire moyen dans la province a atteint, en 1949, $\$ 1.24$ l'heure - à comparer à $\$ 1.16$ pour $1947-48$, à $\$ 1.01$ pour 1946-47 et à .614 pour l'année qui a précédé la guerre, soit 1938-39. Les opérations majeures comprennent le travail des piqueurs, monteurs, tireurs de bouts, tailleurs travaillant du cuir à semelles, fraiseurs de lisses et lustreurs. Voici un tableau intéressant qui indique la moyenne annuelle des salaires payés aux ouvriers de la classe 1 prévue au décret, par quatorze (14) manufactures types de la province pour la dernière décade:

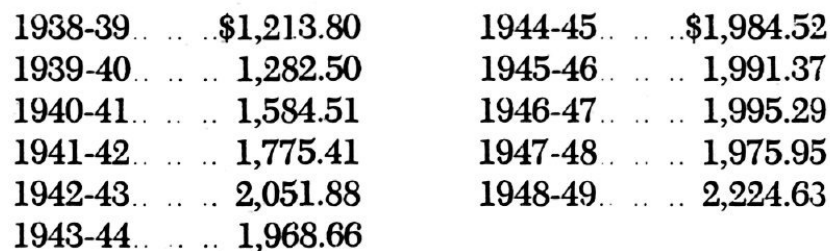

On se rendra compte que le salaire a pratiquement doublé pour ces ouvriers qualifiés.

Ajoutons que l'industrie accorde maintenant aux salariés deux (2) semaines de congés payés dont l'un au cours de l'été et l'autre durant la période des fêtes.

\section{Conclusions}

Il est intéressant de tirer quelques conclusions des faits que nous avons recueillis de ce rapport annuel.

1. L'industrie de la chaussure demeure principalement québecoise, la valeur de la production dans notre province atteignant $60.7 \%$ pour l'année 1948. En 1937 ce pourcentage marquait 60.5.

On remarque la même stabilité dans la province d'Ontario; la valeur de la production pour 1948 y est de $35.3 \%$ par rapport au total; en 1937 , elle était de $35.5 \%$.

De part et d'autre les positions ont été gardées et l'industrie de la chaussure n'a pas réussi à s'implanter dans les provinces de l'ouest ou de l'est où la production totale atteint moins de $5 \%$ de l'ensemble.

Cette stabilité laisse à conclure que les prix de revient n'ont pas été accrus de façon disproportionnée soit dans le Québec, soit dans l'Ontario, autrement on aurait constaté un foyer d'appel dans l'une ou l'autre de ces provinces. Le salaire, qui constitue un élément primordial du prix de revient 
dans l'industrie de la chaussure, a donc été stabilisé grâce aux décrets pris en exécution de la Loi de la convention collective.

2. Dans la province de Québec elle-même s'est établi un équilibre entre les Zones I, II et III. Aucune zone ne semble avoir subi de régression notable dans ses activités. En 1949, la Zone I comptait 88 établissements par rapport à 64 en 1937; la Zone II, 34 établissements par rapport à 33; la Zone III, 85 établissements par rapport à 52 .

Seule la Zone II (Québec) ne paraît pas s'être développée quant au nombie des ateliers. A noter que la zone rurale est passée de 52 établissements à 85 et que la proportion arithmétique y est plus importante que dans la Zone I. Il faut conclure de là que les écarts de salaires de $5 \%$ et $15 \%$ entre la Zone I et les Zones II et III ont été établis avec prudence par les parties contractantes à la convention collective. L'industrie rurale, loin de disparaître comme on l'avait d'abord cru en certains milieux, a réussi à se développer dans plus d'une trentaine de centres.

3. Il y a 9 petites industries à Montréal ( 1 à 10 salariés), 6 à Québec et 30 dans les autres centres. Il est évident que les petites industries se développent davantage dans les petits centres.

Quant à l'industrie moyenne (10 à 25 salariés), elle garde un développement presque identique pour chaque zone: 15 établissements à Montréal, 5 à Québec et 18 dans les autres centres.

Quant à la troisième catégorie d'établissements ( 25 salariés et plus), elle est représentée par 64 ateliers à Montréal, 23 à Québec et 37 dans les petits centres.

Il y a donc à noter que l'industrie à forte production peut se développer aussi facilement dans les municipalités rurales ou à population moyenne que dans les grands centres. Le décret ne semble avoir eu aucun effet malfaisant à cet égard; il paraît, au contraire, qu'il a permis à l'entreprise privée de se développer au goût de ses initiatives.

$\mathrm{Si}$, d'une part, les grandes villes bénéficient d'un marché du travail plus abondant, de la proximité des sources des matières premières et de la machinerie, des facilités de transport, de communications téléphoniques moins dispendieuses, d'autre part, les petits centres sont compensés par l'écart de $15 \%$ dans les taux de salaires par rapport à la Zone $I$ et, il faut le noter, par une plus grande stabilité de main-d'oeuvre.

4. Les négociations collectives et les commissions mixtes sont devenues, depuis plus d'une décade, des coutumes agréées dans l'industrie de la chaussure. Le résultat est que l'industrie a connu une grande paix sociale. Représentants patronaux et ouvriers se rencontrant chaque mois pour discuter leurs problèmes communs, ont su mieux se connaître et, se connaissant mieux, ont pu résoudre pratiquement toutes les difficultés d'ordre économique et social.

Fait à noter, la présence du syndicalisme ouvrier a obligé les employeurs à faire l'unité dans leur propre syndicalisme; c'est pour cette raison qu'ils ont établi l'Association patronale des manufacturiers de chaussures du Québec, laquelle réunit presque tous les manufacturiers.

5. Les taux fixés par le décret, on le constatera, sont réellement des minima qui sont souvent dépassés. Ainsi, pour les tailleurs de cuir, le salaire minimum horaire prévu par le décret est de $\$ 1.045$ pour la Zone I, de .99 pour la Zone II et de .915 pour la Zone III (salariés à la pièce). Le salaire horaire réel moyen pour ces mêmes ouvriers a été de $\$ 1.23$ pour la Zone I, $\$ 1.12$ pour la Zone II et $\$ 1.048$ pour la Zone III. Nous pourrions compléter la preuve en utilisant la documentation du Comité paritaire. On peut conclure de là que le décret n'établit pas, en fait, de minima absolus; ceux-ci sont toujours dépassés grâce à l'augmentation de la productivité des ouvriers qui, par le truchement du travail à la pièce, bénéficient de primes au rendement.

6. On voit également que la durée moyenne du travail s'établit à 32.5 heures pour l'année 194849. Le salaire garanti, s'il est jamais demandé, ne pourrait guère dépasser la rémunération d'une semaine de 30 heures dans cette industrie. Comme toute moyenne d'ordre statistique, celle-ci ne donne pas l'image réelle de la situation dans l'industrie; celle-ci connaît des à-coups, des hausses et des baisses de production qui sont provoqués par les aléas de la température, les caprices de la mode et la dépendance des styles canadiens vis-à-vis des styles américains.

Somme toute, nous pouvons conclure que l'industrie de la chaussure, en autant qu'il s'agit des relations de travail, connaît la stabilité dans notre province grâce à la collaboration des organisations patronales et ouvrières. On a pratiquement réussi, tout en faisant la part des choses, à obtenir la paix sociale; une saine technique des relations industrielles s'est établie après un dur apprentissage et elle donne aujourd'hui aux hommes de bonne volonté qui l'ont développée des résultats dont nous nous réjouissons avec eux. 